\title{
Surface Plasmon Enhanced Light-Emitting Diode
}

\author{
Jelena Vučković, Marko Lončar, and Axel Scherer
}

\begin{abstract}
A method for enhancing the emission properties of light-emitting diodes, by coupling to surface plasmons, is analyzed both theoretically and experimentally. The analyzed structure consists of a semiconductor emitter layer thinner than $\lambda / 2$ sandwiched between two metal films. If a periodic pattern is defined in the top semitransparent metal layer by lithography, it is possible to efficiently couple out the light emitted from the semiconductor and to simultaneously enhance the spontaneous emission rate. For the analyzed designs, we theoretically estimate extraction efficiencies as high as $37 \%$ and Purcell factors of up to 4.5. We have experimentally measured photoluminescence intensities of up to 46 times higher in fabricated structures compared to unprocessed wafers. The increased light emission is due to an increase in the efficiency and an increase in the pumping intensity resulting from trapping of pump photons within the microcavity.
\end{abstract}

Index Terms-Finite-difference time-domain methods, light-emitting diodes, optics at surfaces, spontaneous emission, surface plasmons.

\section{INTRODUCTION}

$\mathbf{F}$ OR years, a significant amount of scientific work has been focused on ways of improving the extraction efficiency of light-emitting diodes (LEDs). Many interesting approaches have been proposed to accomplish this, such as the use of thin light-emitting layers with surface texturing [1], resonant cavities [2], photon recycling [3], or output coupling through surface plasmons excited at corrugated metal surfaces [4]. External quantum efficiencies of $31 \%$ were reported by employing reflection from a bottom metal mirror together with a textured top semiconductor surface [5].

Apart from efforts to extract as much light as possible from a semiconductor device, it is also possible to enhance the light emission rate within a semiconductor. This approach is based on Purcell's prediction in 1946 that the radiation rate of an atom placed within a wavelength-sized cavity can be changed [6]. A fivefold enhancement of spontaneous emission was recently measured in a semiconductor optical microcavity at low temperatures [7], and Yablonovitch and coworkers demonstrated [8] that Purcell factors of about 55 can be achieved when a $\mathrm{InGaN} / \mathrm{GaN}$ quantum well $(\mathrm{QW})$ is positioned close to a thin silver layer.

In order to build an ideal, highly efficient light-emitting diode (LED), it is desirable to improve the extraction efficiency and simultaneously enhance the spontaneous emission rate. A 15 -fold emission intensity enhancement, with Purcell factor $F_{p}=2$ was observed in 2-D periodic thin film photonic crystals [9]. On the

Manuscript received December 27, 1999; revised July 10, 2000. This work was supported by the Air Force Office of Scientific Research (FOSR) under Contract AFS-5XF49620-1-044-SC.

The authors are with the Department of Electrical Engineering, California Institute of Technology, Pasadena, CA 91125 USA (e-mail: jela@ caltech.edu).

Publisher Item Identifier S 0018-9197(00)08144-6.
TABLE I

LAYERS OF THE GROWN WAFER

\begin{tabular}{c|c|c}
\hline & layer & thickness $[\mathrm{nm}]$ \\
\hline 8 & $\mathrm{p}-\mathrm{GaAs}$ cap & 10 \\
\hline 7 & $\mathrm{p}-A l_{x} G a_{1-x} A s, x<0.3$ & 20 \\
\hline 6 & undoped GaAs & 10 \\
\hline 5 & undoped $I n_{0.2} G a_{0.8} A s \mathrm{QW}$ & 8 \\
\hline 4 & undoped GaAs & 10 \\
\hline 3 & $\mathrm{n}-A l_{x} G a_{1-x} A s, x<0.3$ & 20 \\
\hline 2 & n-GaAs cap & 10 \\
\hline 1 & undoped AlAs (sacrificial layer) & 100 \\
\hline 0 & undoped GaAs substrate & - \\
\hline
\end{tabular}

other hand, Barnes [10] recently discussed a potentially highly efficient LED consisting of a metal clad dielectric microcavity with periodic texturing of one of metal layers. He noted that coupling to surface plasmon modes could improve light emission properties of the device. Our work, presented in this paper, is the first experimental demonstration of this novel method for enhancing the light emission from LEDs.

In Section II of this article, we describe the procedure that we developed for fabricating metal clad semiconductor microcavities with periodic texturing of the top, semitransparent metal layer. In Section III, we present the theoretical analysis of their band structures, electromagnetic fields, external and extraction efficiencies, Purcell factors, as well as the calculated transmission of pump power through the top surface and the increase in the pumping intensity resulting from trapping of pump photons within the microcavity. Finally, in Section IV, we present and discuss results of photoluminescence (PL) measurements from fabricated structures.

\section{FABRICATION}

Design of the grown wafer is shown in Table I. Wafer was designed for fabrication of electrically pumped devices and p- and n-doped layers were included. Layers $2-8$ form the membrane that will be lifted off and sandwiched between two metal layers. The total membrane thickness is approximately $90 \mathrm{~nm}$. The emission from the InGaAs/GaAs QW is centered at $986 \mathrm{~nm}$, which corresponds to conduction-to-heavy hole band transitions (C-HH). There is also a peak at $930 \mathrm{~nm}$, corresponding to conduction-to-light hole band transitions (C-LH), which becomes more prominent at high pumping levels. $\mathrm{C}-\mathrm{HH}$ transitions couple to electric fields polarized in the QW plane $(x-z$ plane). On the other hand, C-LH transitions couple twice as strongly to electric fields with polarization perpendicular to the QW plane ( $y$ direction) than to those polarized in the QW plane [11]. In the classical spontaneous emission model, C-HH transitions are represented with parallel dipoles, 

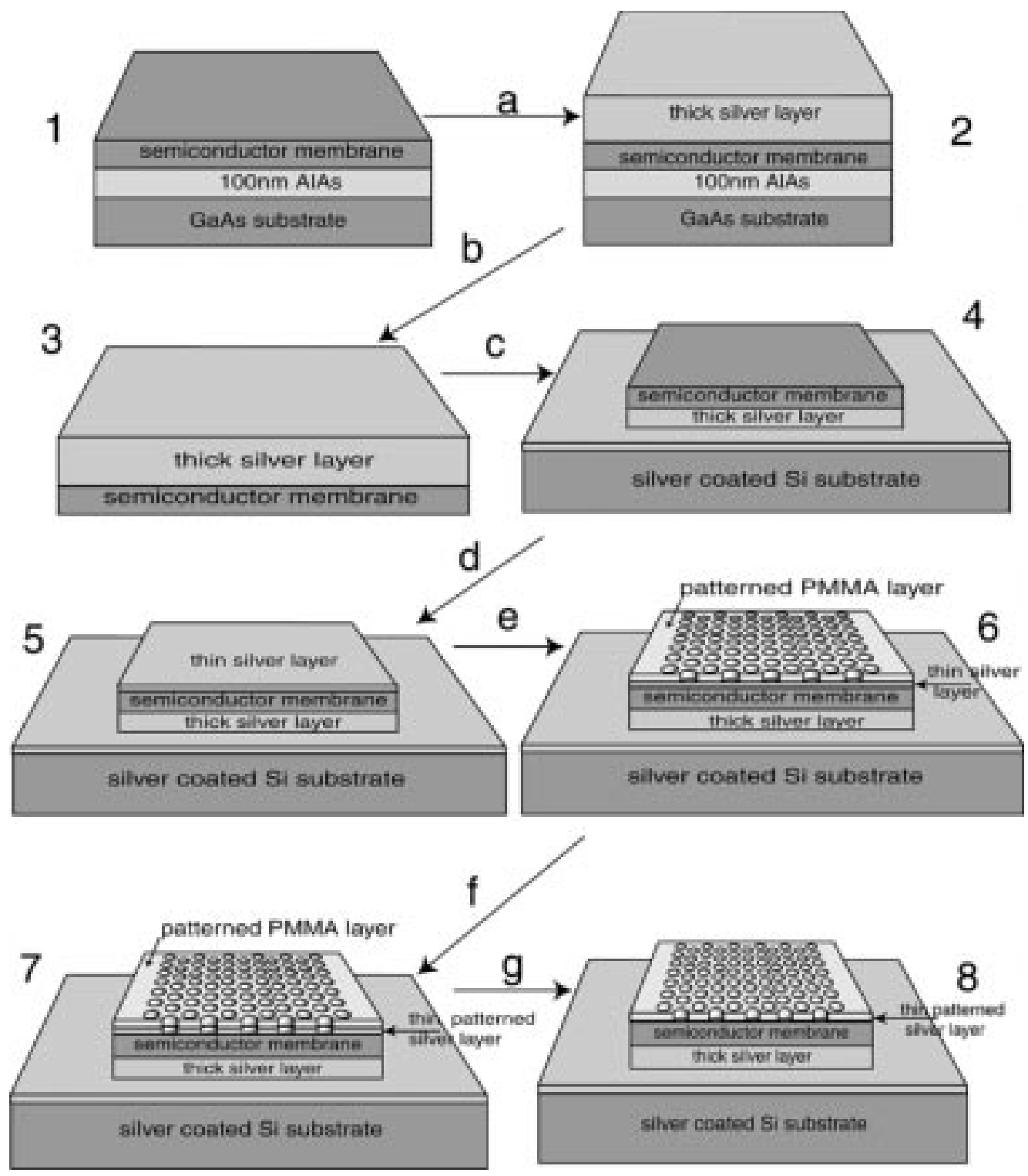

Fig. 1. Fabrication procedure: (a) thick silver layer deposition; (b) epitaxial liftoff; (c) Van der Waals bonding onto silver coated silicon substrate; (d) thin silver layer deposition; (e) PMMA deposition and patterning using e-beam lithography; (f) pattern transfer to thin silver layer using $\mathrm{Ar}^{+}$ion milling; and (g) PMMA removal.

while C-LH transitions are represented with both parallel and perpendicular dipoles, weighted by factors of $1 / 3$ and $2 / 3$, respectively.

The fabrication procedure is described in Fig. 1. First, we deposit a thick silver mirror $(d>1.5 \mu \mathrm{m})$ on top of the grown wafer [step (a)]. This metal layer is also used as a mechanical support during the membrane liftoff. Then, we remove the membrane from its substrate by dissolving the sacrificial AlAs layer in $8.2 \%$ hydrofluoric acid (HF) diluted in water [step (b)]. HF attacks AlAs very selectively over $\mathrm{Al}_{x} \mathrm{Ga}_{1-x} \mathrm{As}$ for $x<0.4$ [12]. The lifted-off membrane (layers 2-8) with the thick silver layer on top is then Van der Waals bonded [12] onto a silver coated silicon wafer and the silver on the lift-off film bonds to the silver coated on the silicon support wafer [step (c)]. Another 20-40-nm-thick silver layer is then deposited on top of the $n$-GaAs cap [step (d)]. A 100-nm-thick film of high molecular weight PMMA (polymethylmethacrylate) is then spun on top of the thin metal layer and subsequently baked on a hot plate at $150{ }^{\circ} \mathrm{C}$ for 20 min. A desired pattern is beamwritten on the PMMA by electron beam lithography in a Hitachi S-4500 electron microscope [step (e)]. The resulting patterns are approximately $50 \mu \mathrm{m}$ $\times 50 \mu \mathrm{m}$ in size, and the exposed PMMA is developed in a 3:7 solution of 2-ethoxyethanol:methanol for $30 \mathrm{~s}$. Then, the pattern is transferred into the top semitransparent metal layer using $\mathrm{Ar}^{+}$ion milling at a beam voltage of $1500 \mathrm{~V}$ [step (f)]. Finally, the remaining PMMA may be dissolved in acetone [step (g)]. A corresponding SEM picture, showing the top view of a fully processed wafer, is given in Fig. 2, where light areas correspond to regions where silver was removed.

The structure shown in Fig. 1.1 is the unprocessed wafer and the one in Fig. 1.4 is referred to as the half-processed wafer. Fig. 1.5 represents the unpatterned metal clad microcavity, and finally, the structure shown in Fig. 1.8 is the fully processed one. 


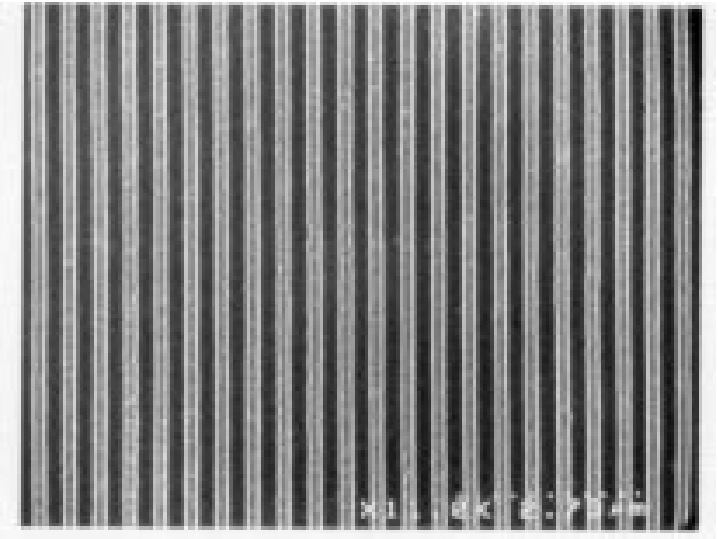

Fig. 2. Fabricated pattern in the top silver layer. Light areas correspond to regions where silver was removed during the $\mathrm{Ar}^{+}$ion milling process.

\section{THEORY}

\section{A. FDTD Analysis of Metallic Structures at Optical Frequencies}

The finite-difference time-domain (FDTD) method is used to theoretically analyze electromagnetic fields within metal clad microcavities. In order to accurately model metals at optical frequencies, it is necessary to make some changes [13], [14] to the standard Yee's FDTD scheme [15]. Electromagnetic fields in metals are described by adding a current term $(\vec{J})$ to Maxwell's curl equations (Drude model)

$$
\begin{aligned}
\vec{\nabla} \times \vec{H} & =\epsilon_{\infty} \frac{\partial \vec{E}}{\partial t}+\vec{J} \\
\vec{\nabla} \times \vec{E} & =-\mu_{0} \frac{\partial \vec{H}}{\partial t} \\
\frac{\partial \vec{J}}{\partial t}+\nu \vec{J} & =\epsilon_{0} \omega_{p}^{2} \vec{E}
\end{aligned}
$$

where $\omega_{p}$ is the plasma frequency of a metal and $\nu$ is the corresponding damping rate. We assume that the metal is silver with parameters $\hbar \omega_{p}=8.8 \mathrm{eV}$ (i.e., $\lambda_{p}=140 \mathrm{~nm}$ ), $\hbar \nu=0.05 \mathrm{eV}$, and $\epsilon_{\infty}=\epsilon_{0}$. In part of our FDTD calculations, we neglect metal absorption losses by applying $\nu=0$. Nonmetallic regions are described with standard Maxwell curl equations

$$
\begin{aligned}
& \vec{\nabla} \times \vec{H}=\epsilon(\vec{r}) \frac{\partial \vec{E}}{\partial t} \\
& \vec{\nabla} \times \vec{E}=-\mu_{0} \frac{\partial \vec{H}}{\partial t}
\end{aligned}
$$

The FDTD method consists, basically, of the discretization of (1)-(5) in space and time [16]. The reader is referred to [17] for the detailed description of the FDTD calculation of band diagrams and filtering of electromagnetic fields for a mode of interest. Depending on the problem, different boundary conditions are applied to boundaries of the computational domain, such as the Mur's absorbing boundary conditions (Mur's ABC [18]) or Bloch boundary conditions. The spatial discretization step is critical in this case, keeping in mind that the penetration depth of the electromagnetic field into metals can be of the order of only tens of nanometers. This implies that large amounts of memory are required for computation unless variable cell sizes

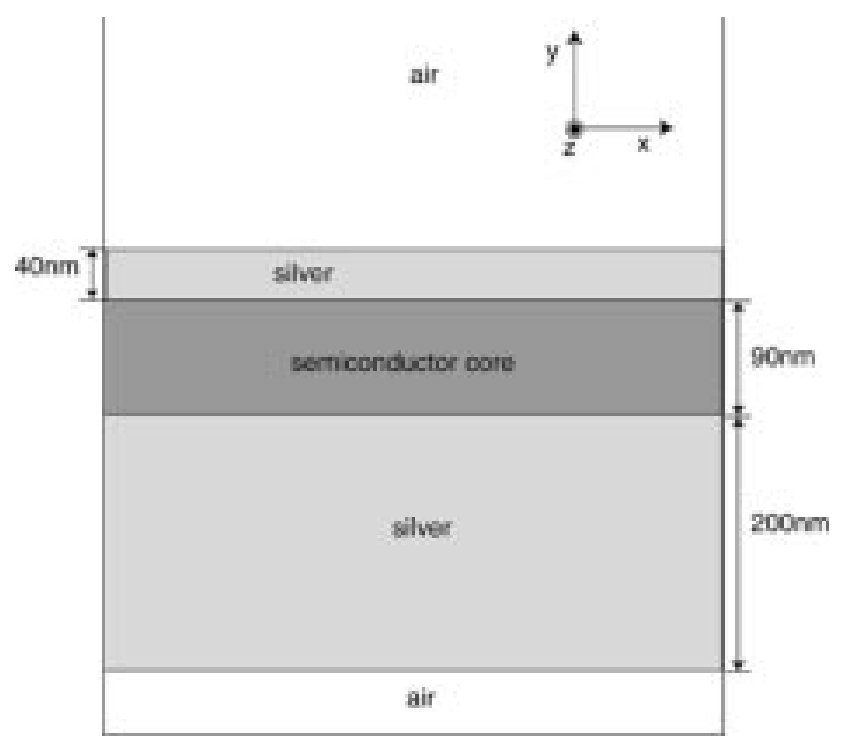

Fig. 3. A metal clad microcavity analyzed using the FDTD method. The structure is infinite in the $z$ direction. Mur's absorbing boundary conditions are applied to boundaries in the $y$ direction and Bloch boundary conditions are applied to boundaries in the $x$ direction.

are used. We have performed 2-D analyses to design our structures, with discretization steps of $3 \mathrm{~nm}$ (unless noted otherwise).

\section{B. Electromagnetic Fields and Band Diagrams of Unpatterned Metal Clad Microcavities}

First, we analyze the band diagram of the metal clad microcavity shown in Fig. 3. Parameters of the analyzed structure are chosen so that they correspond to geometries which we have fabricated and measured. The semiconductor membrane was $90 \mathrm{~nm}$ thick, with a refractive index of $n=3.5$. The top semitransparent silver layer was $40 \mathrm{~nm}$ thick, and the bottom silver layer was $200 \mathrm{~nm}$ thick. Absorption losses in silver were not included in any of the band diagram calculations, since if the bands are too lossy, it is difficult to recover them from spectra obtained in FDTD analyses. In the wavelength range of our interest $(986 \mathrm{~nm})$, the imaginary part of the silver dielectric constant is still much smaller than its real part, and positions of bands can be determined approximately by assuming that $\nu=0$. The analyzed structure is surrounded by air on top and bottom and the slab is infinite in the horizontal plane. We assume that the structure is "periodic" in the $x$ direction with an artificial periodicity of $50 \mathrm{~nm}$ and analyze only one unit cell. Mur's ABC are applied to boundaries in the $y$ direction, while Bloch boundary conditions are applied to boundaries in the $x$ direction.

$P$-polarized (TM) light has $x$ and $y$ components of the electric field and the $z$ component of the magnetic field, while $s$-polarized (TE) light has $x$ and $y$ components of the magnetic field and the $z$ component of electric field. The band diagram for the structure shown in Fig. 3 is shown in Fig. 4. The discretization step used in the FDTD calculation is equal to $1 \mathrm{~nm}$. We observed that the increase in the discretization step (to $3 \mathrm{~nm}$ ) influenced only the $\mathrm{TM}_{0}$ band: the band shifted upwards in frequency at large $k_{x}$ values. The splitting between the long- and short-range coupled SPP branches [10] of the top silver layer 


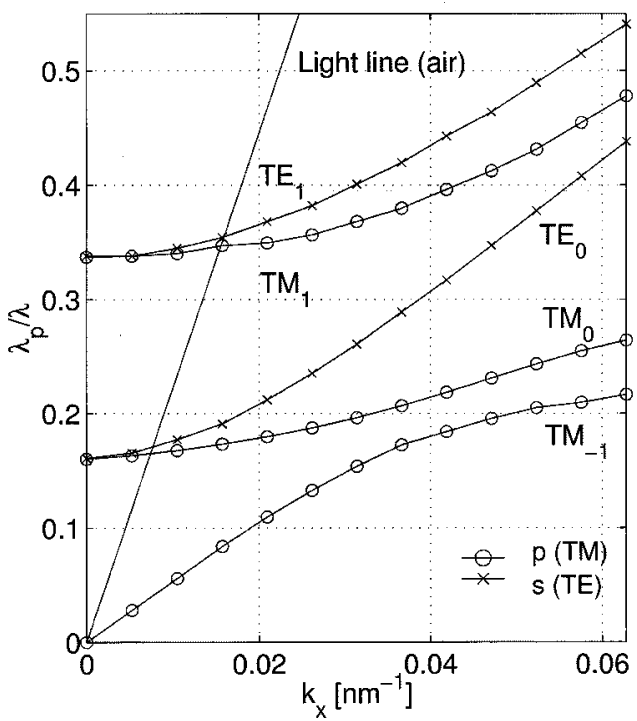

Fig. 4. Band diagram of the unpatterned (flat) metal clad microcavity. Silver layers are 40 and $200 \mathrm{~nm}$ thick and the semiconductor core thickness is $90 \mathrm{~nm}$. Absorption losses in silver were not included.

was not observed for the semitransparent silver layer thickness used in FDTD calculations $(40 \mathrm{~nm})$ and for the used frequency resolution of $2.3 \cdot 10^{-3} \lambda_{p} / \lambda$. Let us analyze electromagnetic fields in the middle of the membrane, where the $\mathrm{QW}$ is located in real structures. The $\mathrm{TM}_{-1}$ mode has a very weak parallel $\left(E_{x}\right)$ and a strong perpendicular $\left(E_{y}\right)$ electric field component there, as shown in Fig. 5. It follows that perpendicular dipoles positioned in the QW couple strongly to this mode. On the other hand, the $\mathrm{TM}_{0}$ mode has a strong parallel $\left(E_{x}\right)$ and a weak perpendicular $\left(E_{y}\right)$ electric field component in the middle of the membrane, as can be seen in Fig. 6. (Fig. 6 represents the $\mathrm{TM}_{0}$ mode at cutoff where it is degenerate to the $\mathrm{TE}_{0}$ mode and the $y$ component of its electric field is equal to zero.) Furthermore, the $\mathrm{TE}_{0}$ mode has only its parallel $\left(E_{z}\right)$ component of electric field not equal to zero. Therefore, parallel dipoles in the $\mathrm{QW}$ couple strongly to $\mathrm{TM}_{0}$ and $\mathrm{TE}_{0}$ modes.

Using the 1-D finite difference method, we evaluated the cutoff frequency of $\mathrm{TE}_{0}$ and $\mathrm{TM}_{0}$ modes when absorption losses in both metal and semiconductor were included, and concluded that it was positioned at $\lambda_{p} / \lambda=0.15$ instead of 0.16 , as in the lossless band diagram shown in Fig. 4. Absorption losses also reduce the quality factors of modes. Using the FDTD method, we evaluated $Q$-factors corresponding to several $\left(k_{x}, \lambda_{p} / \lambda\right)$ points in the band diagram (including metal absorption) and they were in the range between $5-7$. Therefore, for lossy bands, we cannot talk about an abrupt cutoff at $\lambda_{p} / \lambda=0.15$. Instead, the cutoff is gradual and extends from about $\lambda_{p} / \lambda=0.13$ to 0.15 .

In normalized units, the $\mathrm{C}-\mathrm{HH}$ transitions peak at $986 \mathrm{~nm}$ which corresponds to $\lambda_{p} / \lambda=0.14$. From the previous discussion, it follows that these transitions couple mostly to $\mathrm{TE}_{0}$ and $\mathrm{TM}_{0}$ modes in the gradual cutoff. Their coupling to the $\mathrm{TM}_{-1}$ mode is very weak. On the other hand, the C-LH transitions peak at $930 \mathrm{~nm}$ corresponds to $\lambda_{p} / \lambda=0.15$ and this emission couples strongly to the $\mathrm{TM}_{-1}$ mode. However, one third of C-LH

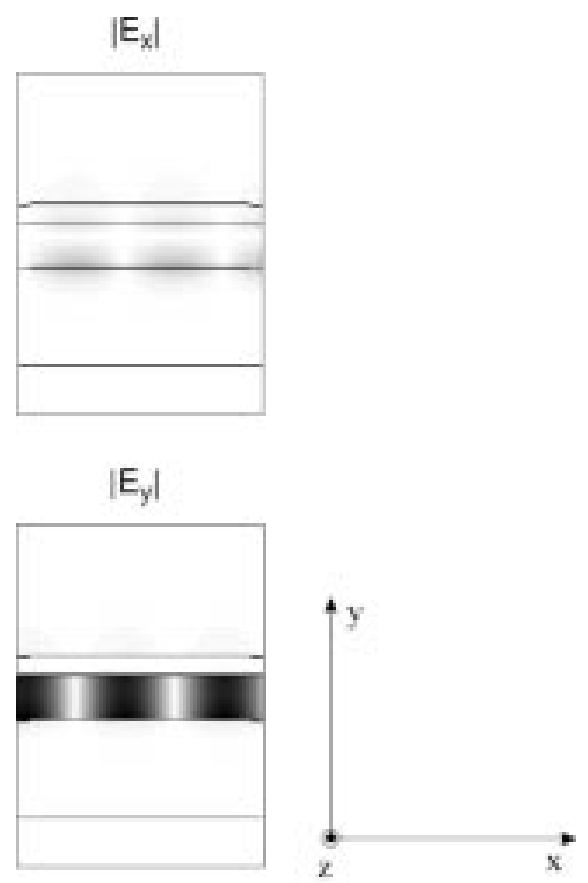

$\left|\mathrm{B}_{z}\right|$

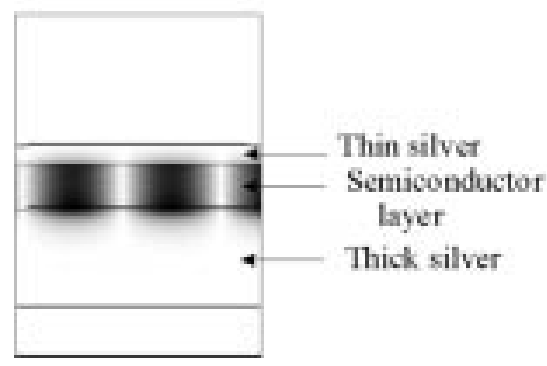

Fig. 5. Intensities of electric and magnetic field components for the $\mathrm{TM}_{-1}$ mode, with $\lambda_{p} / \lambda=0.084$ and $k_{x}=0.0157 \mathrm{~nm}^{-1}$. The analyzed structure has the same parameters as the one whose band diagram is shown in Fig.4.

dipole transitions are in plane and they can couple to $\mathrm{TE}_{0}$ and $\mathrm{TM}_{0}$ modes.

\section{Electromagnetic Fields and Band Diagrams of Metal Clad Microcavities with a Patterned Semitransparent Metal Layer}

Perpendicular dipoles in metal clad microcavities couple strongly to the $\mathrm{TM}_{-1}$ mode [19] located below the light line, as shown in the band diagram in Fig. 4. Therefore, their emission cannot be extracted outside an unpatterned microcavity. Usually, the $\mathrm{TM}_{-1}$ mode is considered a loss mechanism in metallic structures. However, we can modify the band diagram and bring parts of the $\mathrm{TM}_{-1}$ branch above the light line by patterning of the top semitransparent metal layer. The introduction of periodicity into one of metallic layers dramatically changes the band diagram shown in Fig. 4. The band diagram is folded back into its first Brillouin zone at the edges of which the band gap for surface plasmon waves appears [10]. This means that bands previously located below the light line can now be brought above it. The effect of the periodic patterning can also be analyzed using the 2-D FDTD. We introduce a 1-D grating consisting of stripes (infinite in the 


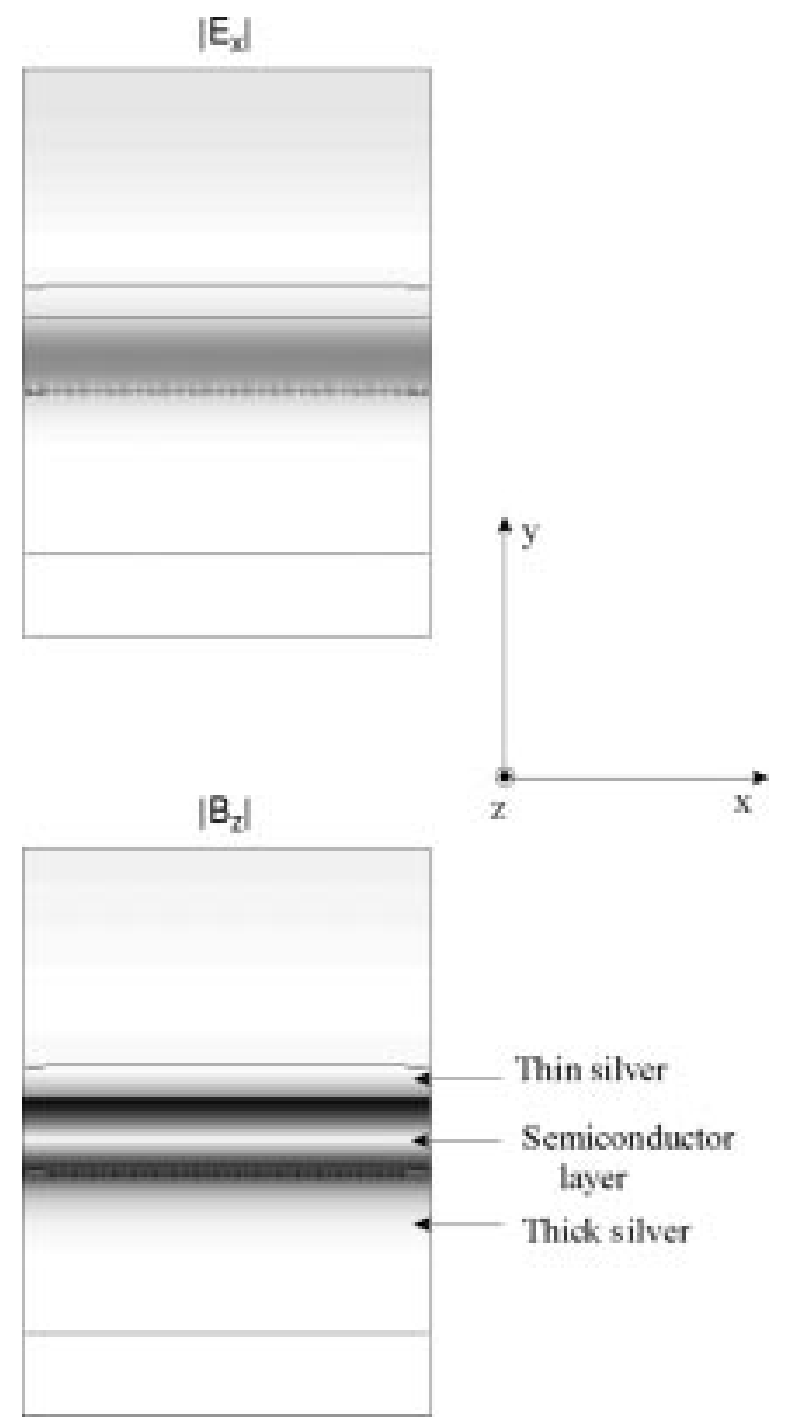

Fig. 6. Intensities of the $x$ component of the electric field and the $z$ component of the magnetic field for the $\mathrm{TM}_{0}$ mode, with $\lambda_{p} / \lambda=0.16$ and $k_{x}=0$. $E_{y}=0$ for this mode. The analyzed structure has the same parameters as the one whose band diagram is shown in Fig. 4.

$z$ direction) within the top metal layer. The pattern is formed by periodically modulating the top metal layer thickness between $40-0 \mathrm{~nm}$. When the top layer is modulated between $20-0 \mathrm{~nm}$, the results are approximately the same. We analyze three structures, with the same parameters as those that we fabricated and measured. Their grating periodicities are 250, 480, and $650 \mathrm{~nm}$, with a 160-nm-wide gap between silver stripes. The schematic diagram of these structures is shown in Fig. 7 and the calculated band diagrams for TE and TM polarizations are shown in Figs. 8-10.

The indicator of how strongly the grating changes the band diagram of an unpatterned metal clad microcavity is the size of the band gap that opens at the edge of the Brillouin zone. Let us first consider band diagrams for the TM polarization. Clearly, in the frequency range of our interest $\left(\lambda_{p} / \lambda \approx 0.14\right)$ only the grating with a periodicity of $250 \mathrm{~nm}$ strongly affects properties of the structure. We expect that this grating will extract the emission of perpendicular dipoles, while the other two analyzed structures will not show any significant improvement over an

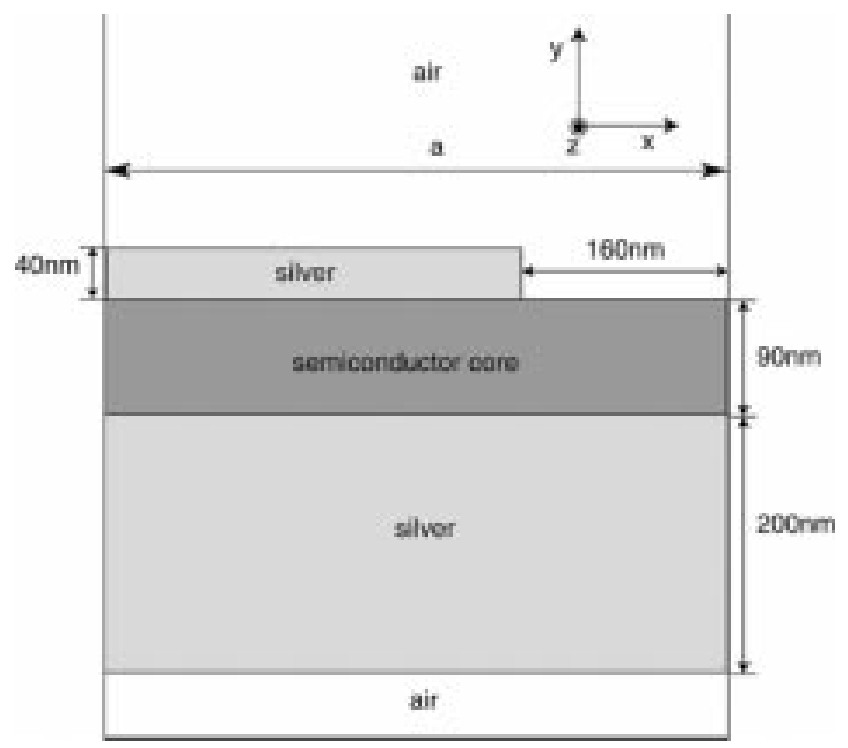

Fig. 7. The structure with a grating defined in the top semitransparent layer analyzed using the FDTD method in order to study the effect of metal patterning. Mur's absorbing boundary conditions are applied to boundaries in the $y$ direction and Bloch boundary conditions are applied boundaries in the $x$ direction. $a$ denotes the grating periodicity.

unpatterned metal clad microcavity. With the frequency resolution of $2.3 \cdot 10^{-3} \lambda_{p} / \lambda$ used in our FDTD calculations, we could not detect a band gap at $k_{x}=0$ and $\lambda_{p} / \lambda \approx 0.14$ for structures with periodicities of 650 or $480 \mathrm{~nm}$. What about the TE polarization? A band with the cutoff frequency around 0.14 appears in TE band diagrams for all structures. As we will see from the electric field distribution, this branch corresponds to the combination of the $\mathrm{TE}_{0}$ mode and the mode that resonates in the gap between silver stripes. For the smaller grating periodicity, gaps between silver stripes are closer to each other and behave as coupled cavities. Therefore, this mode can propagate in the $x$ direction. On the other hand, for the larger grating periodicity (such as $480 \mathrm{~nm}$ ), cavities are decoupled, this mode cannot propagate, and the corresponding TE band is flat. For the upper TE bands, the band gap does not appear at the edges of the Brillouin zone, meaning that the grating has basically no effect there and the corresponding mode is simply the TE mode of the unpatterned structure.

First, we filtered the TM polarized fields with $k_{x}=0$ and $\lambda_{p} / \lambda \approx 0.14$ for the structure with a periodicity of $250 \mathrm{~nm}$, in order to confirm that this metal layer patterning produces the outcoupling of radiation. From the band diagram shown in Fig. 8, we see that there are two modes in the filtered frequency range. The intensities of $x$ and $y$ components of electric field are shown in Fig. 11. By comparison with the fields from Fig. 5, we see that radiation now escapes from the microcavity, even though the gap between silver stripes is smaller than a wavelength. Moreover, the $x$ component of the electric field is not negligible in the middle of the membrane. This means that parallel dipoles positioned there and oriented in the $x$ direction can also couple to this mode. On the other hand, the $E_{y}$ field is still strong within the membrane, which means that perpendicular dipoles still couple to this mode strongly.

Then, we filtered the TM polarized fields with $k_{x}=0$ and $\lambda_{p} / \lambda \approx 0.15$, for the structure with a periodicity of $650 \mathrm{~nm}$. The 


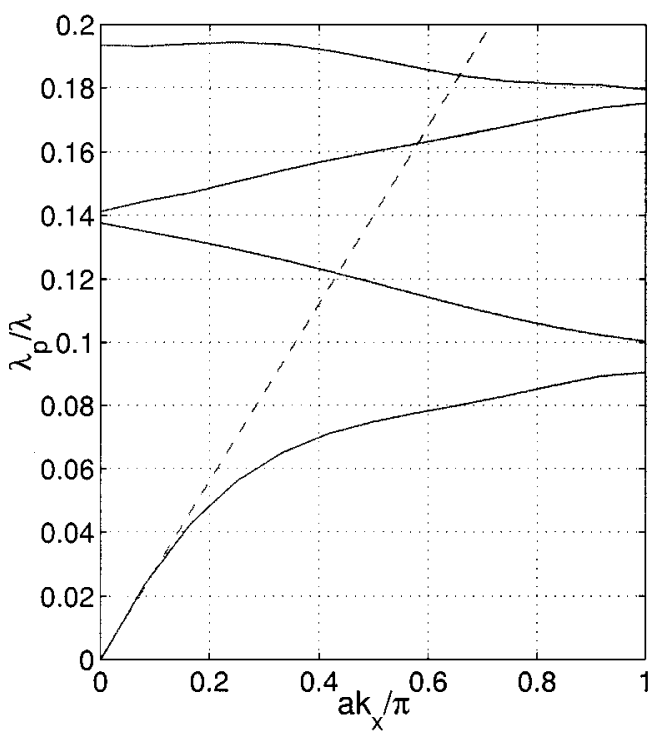

(a)

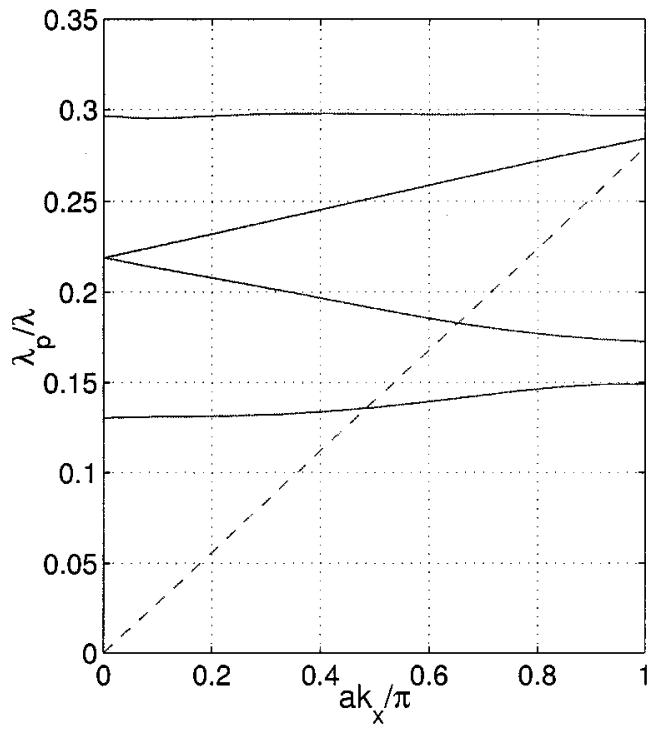

(b)

Fig. 8. Band diagrams of the patterned structure with a periodicity of $a=250 \mathrm{~nm}$ : (a) TM-polarization and (b) TE-polarization. The dashed line corresponds to the light line in air.

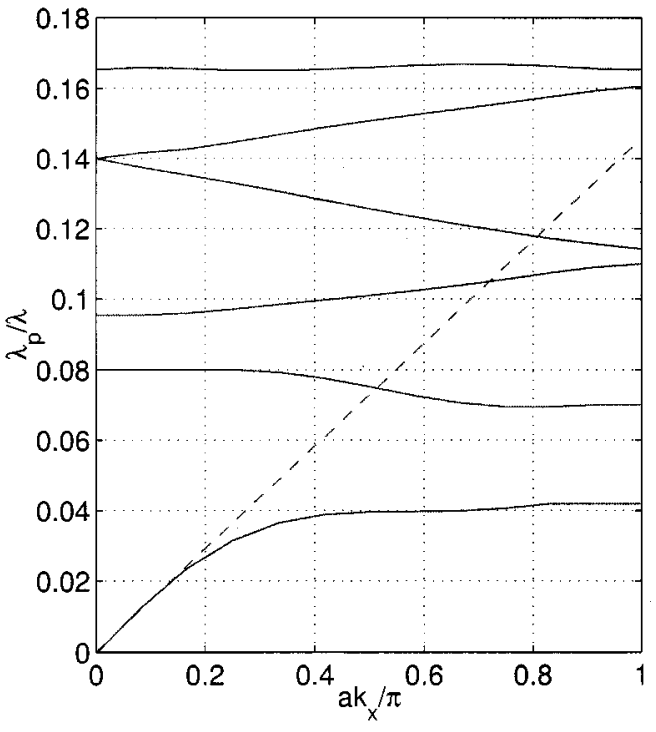

(a)

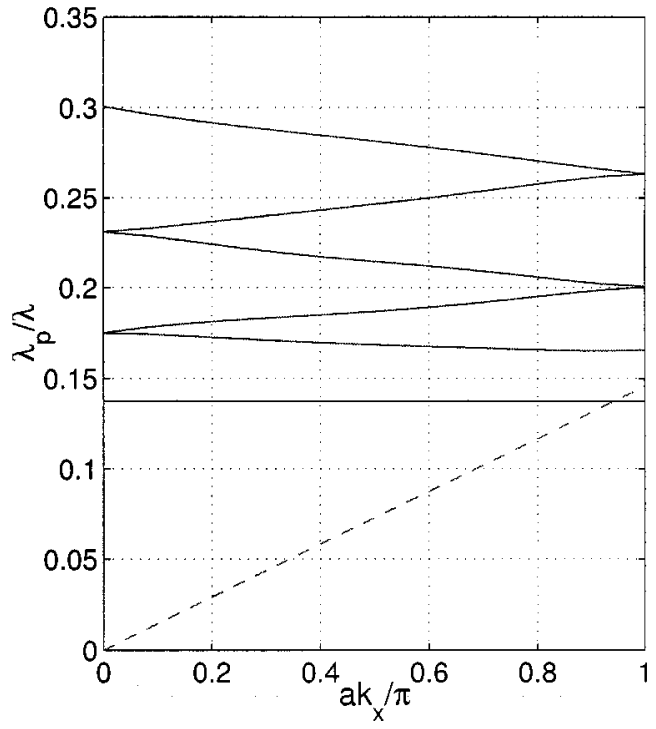

(b)

Fig. 9. Band diagrams of the patterned structure with a periodicity of $a=480 \mathrm{~nm}$ : (a) TM-polarization and (b) TE-polarization. The dashed line corresponds to the light line in air.

electric field intensity patterns are represented in Fig. 12. They look exactly like the $\mathrm{TM}_{-1}$ mode with $k_{x}=6 \pi / a$ of the unpatterned structure, except underneath gaps between silver stripes. The electromagnetic field intensity outside the microcavity is small. This was expected from the band diagram for the TM polarization for this structure, shown in Fig. 10, since we could not detect opening of the band gap in the filtered frequency range.

We also filtered the TE polarized fields with $k_{x}=0$ and $\lambda_{p} / \lambda \approx 0.14$ for the structures with periodicities of 250 and $480 \mathrm{~nm}$. The corresponding $E_{z}$ field distributions are shown in Fig. 13. The mode looks like the $\mathrm{TE}_{0}$ mode that also resonates in the gap between silver stripes, and is radiated out of the cavity through the spacing between stripes. Since the density of these spacings is larger for the structure with a periodicity of $250 \mathrm{~nm}$, the mode will extract the emission out of this cavity more efficiently.

Therefore, the patterning of the top metal layer has a strong effect on properties of both TE and TM band diagrams. By choosing a grating appropriately, such as in the case of the analyzed structure with a periodicity of $250 \mathrm{~nm}$, both the emission of perpendicular and parallel dipoles can be extracted out of the microcavity. 


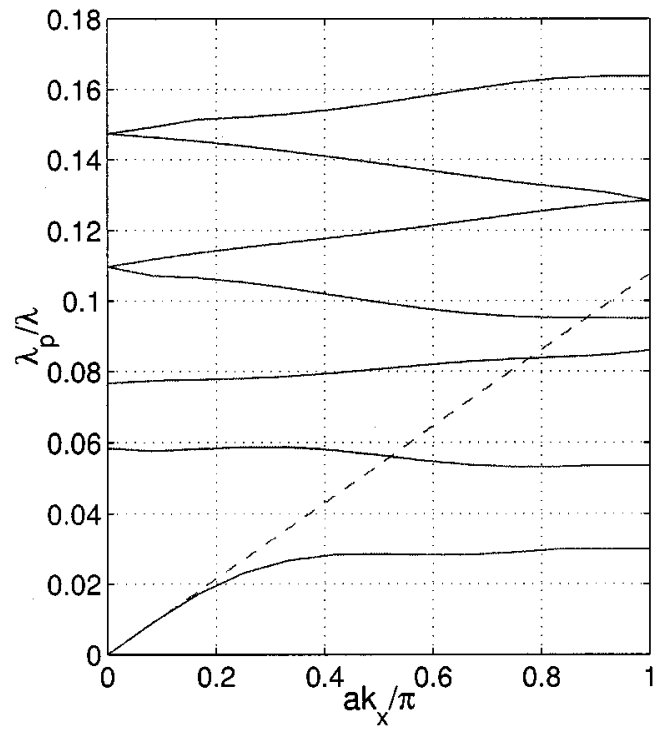

Fig. 10. Band diagram of the structure with a grating periodicity of $650 \mathrm{~nm}$ (TM-polarization only). The dashed line corresponds to the light line in air.

\section{External Efficiency and Extraction Efficiency}

Let us denote by $1 / \tau_{r}$ the rate at which photons are radiated outside the cavity, and $1 / \tau_{\mathrm{mr}}$ the decay rate corresponding to all other mechanisms (such as absorption losses or the excitation of modes that remain trapped within the cavity). The total decay rate is defined as

$$
\frac{1}{\tau}=\frac{1}{\tau_{r}}+\frac{1}{\tau_{\mathrm{nr}}} .
$$

The external efficiency $\left(\eta_{\text {ext }}\right)$ can be expressed as [9]

$$
\eta_{\mathrm{ext}}=\frac{\frac{1}{\tau_{r}}}{\frac{1}{\tau_{r}}+\frac{1}{\tau_{\mathrm{nr}}}}=\frac{\frac{1}{\tau_{r}}}{\frac{1}{\tau}} .
$$

The external efficiency for planar structures without metal patterning can be evaluated using the method described in [20] and [21]. We calculated the external efficiency for the unpatterned metal clad microcavity with a 90-nm-thick semiconductor membrane, as a function of the top, semitransparent silver layer thickness, and for the collection angles of $30^{\circ}$ or $90^{\circ}$ with respect to the normal to the surface. The refractive index used for silver was $n=0.14+6.94 i$. The emitter was a parallel or a perpendicular dipole positioned in the middle of the membrane and oscillating at the wavelength of 986 or $930 \mathrm{~nm}$. The calculated external efficiencies corresponding to perpendicular dipoles are negligible, while the external efficiencies of parallel dipoles are shown in Fig. 14. For the unprocessed wafer, we calculate that $\eta_{\text {ext }}$ corresponding to parallel dipoles positioned $45 \mathrm{~nm}$ from the semiconductor/air interface is $2 \%$ into the $90^{\circ}$ collection angle, or $0.5 \%$ into the $30^{\circ}$ collection angle, both at wavelength of 986 and $930 \mathrm{~nm}$. $\eta_{\text {ext }}$ for perpendicular dipoles in the unprocessed wafer is negligible.
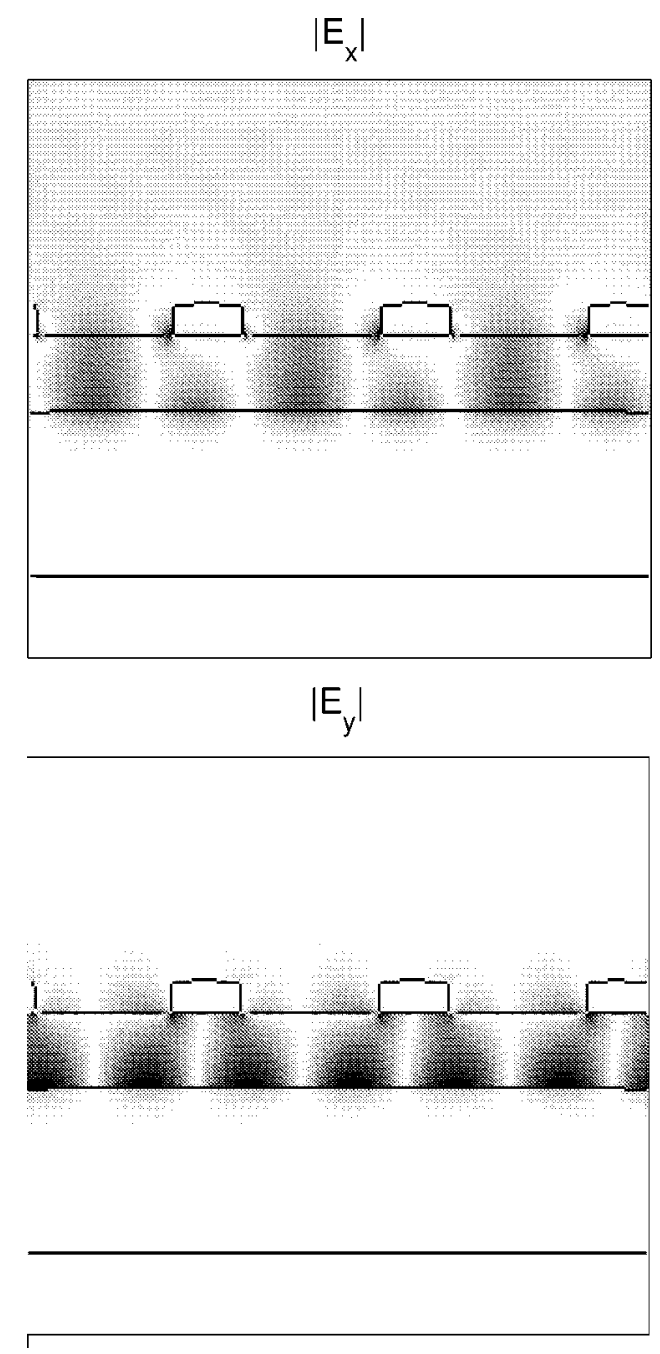

Fig. 11. Intensities of electric field components for the structure with a periodicity of $250 \mathrm{~nm}$. The filtered frequency range was centered around $\lambda_{p} / \lambda=0.14$ and $k_{x}=0$.

For structures with a patterned top metal layer, external efficiencies can be calculated using the 3-D FDTD method, with oscillating dipole sources modeling atomic transitions. However, this computation would require large amounts of memory, as we noted previously. Instead, we can easily estimate, from our 2-D FDTD simulations, the efficiency of light extraction by coupling to some mode. The extraction efficiency $\eta_{x}$ is the probability that a photon radiated into some mode escapes the cavity. The rate at which the electromagnetic field energy is lost from the cavity is described by the radiation quality factor $Q_{r}$

$$
Q_{r}=\frac{\omega_{0} W}{P_{r}}
$$

where $W$ represents the electromagnetic field energy stored in the cavity, $\omega_{0}$ is the radial frequency of a mode, and $P_{r}$ is the power radiated outside the cavity. On the other hand, the nonradiative quality factor $Q_{\mathrm{nr}}$ describes the rate at which the stored electromagnetic energy decreases due to mechanisms other than the radiation outside the cavity

$$
Q_{\mathrm{nr}}=\frac{\omega_{0} W}{P_{\mathrm{nr}}}
$$




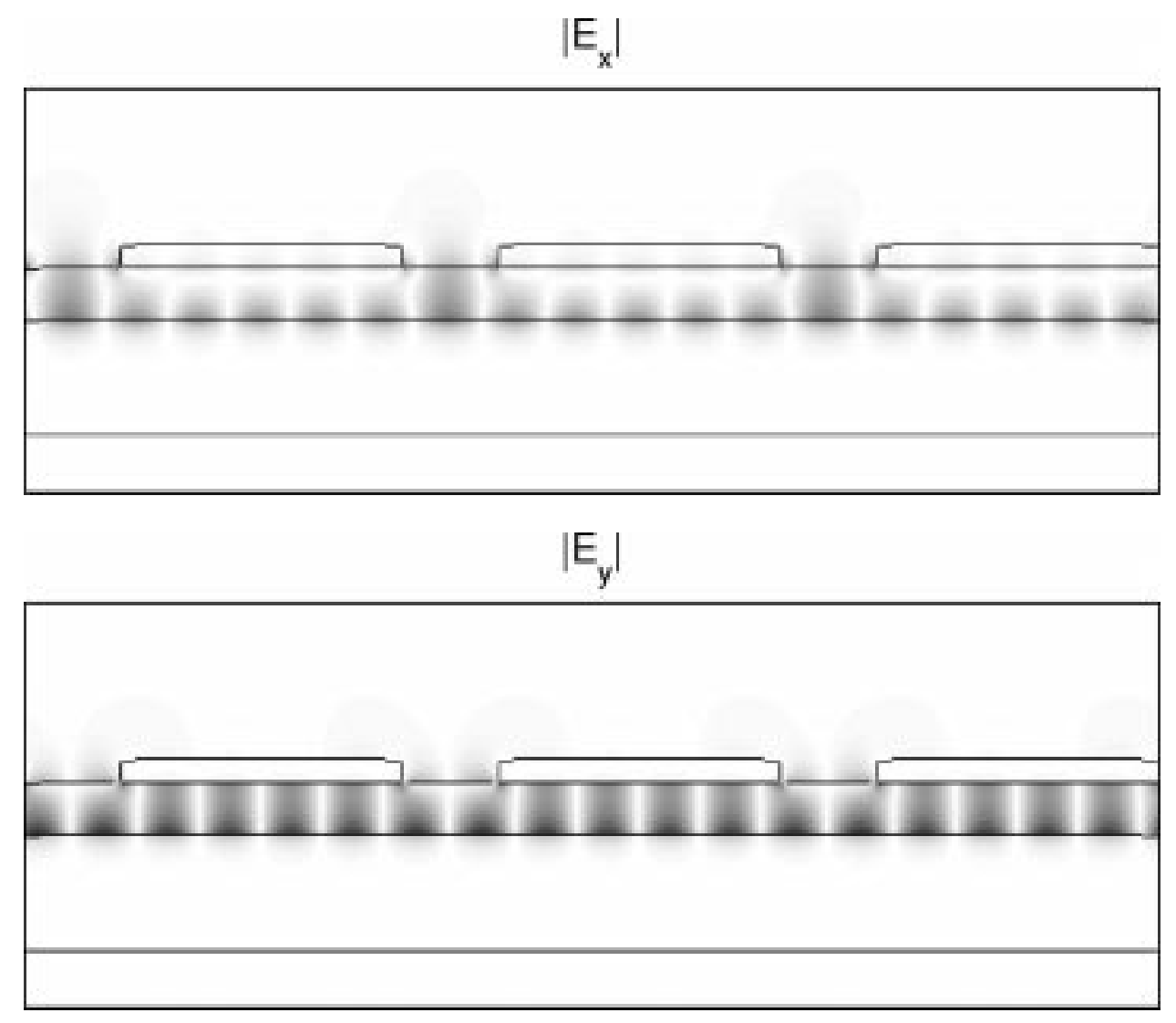

Fig. 12. Intensities of electric field components for the structure with a periodicity of $650 \mathrm{~nm}$. The filtered frequency range was centered around $\lambda_{p} / \lambda=0.15$ and $k_{x}=0$.

where $P_{\mathrm{nr}}$ represents the power lost through these mechanisms. The total quality factor $Q$ is defined as

$$
\frac{1}{Q}=\frac{1}{Q_{r}}+\frac{1}{Q_{\mathrm{nr}}}
$$

and the radiative and nonradiative photon lifetimes are defined as

$$
\begin{aligned}
\tau_{\mathrm{pr}} & =\frac{Q_{r}}{\omega_{0}} \\
\tau_{\mathrm{pnr}} & =\frac{Q_{\mathrm{nr}}}{\omega_{0}}
\end{aligned}
$$

$\eta_{x}$ can be expressed as

$$
\eta_{x}=\frac{\frac{1}{\tau_{\mathrm{pr}}}}{\frac{1}{\tau_{\mathrm{pr}}}+\frac{1}{\tau_{\mathrm{pnr}}}}=\frac{Q}{Q_{r}} .
$$

Clearly, $\eta_{x}$ does not take into account the dependence on the position and orientation of dipole transitions, as well as all possible nonradiative mechanisms.

We calculated $\eta_{x}$ for the structure with a periodicity of $250 \mathrm{~nm}$. The only nonradiative loss mechanism in these calculations was the absorption in metal. For the folded $\mathrm{TM}_{-1}$ mode, whose field distribution is shown in Fig. 11, we calculated $Q_{r}$ between 30 and 50,Q=6 and $\eta_{x}$ between $12 \%$ and $20 \%$. For the TE mode shown in Fig. 13, we calculated $Q_{r}=15, Q=5.5$ and $\eta_{x}=37 \%$. From the band diagram shown in Fig. 8, we can see that the emission in the frequency range from 0.14 to 0.15 (in units of $\lambda_{p} / \lambda$ ) goes into the $30^{\circ}$ escape cone, if coupled to TM modes. However, coupling to TE modes does not improve the directionality and the emission goes into the $90^{\circ}$ cone. For the structures with periodicities of 650 or $480 \mathrm{~nm}$, we estimated that $Q \approx 7$, while the extraction efficiencies corresponding to the folded $\mathrm{TM}_{-1}$ branch were approximately the same as in the unpatterned cavity case. The calculated extraction efficiency corresponding to the first TE band (whose field distribution is shown in Fig. 13) in the structure with a periodicity of $480 \mathrm{~nm}$ is $\eta_{x}=20 \%$. However, only dipoles located directly underneath the gap between silver stripes can couple to this mode, which implies that the external efficiency is at least three times smaller than the calculated $\eta_{x}$. The situation is even worse in the structure with a periodicity of $650 \mathrm{~nm}$. We conclude that the external efficiencies of the structures with periodicities of 480 and $650 \mathrm{~nm}$ are not expected to be better than in the unpatterned structure.

Therefore, for the structure with a periodicity of $250 \mathrm{~nm}$, the extraction efficiency of more than $30 \%$ can be achieved in the presence of a grating. The filtered modes have low quality factors and overlap with both $\mathrm{C}-\mathrm{HH}$ and $\mathrm{C}-\mathrm{LH}$ emission peaks. From the field patterns for the TM mode shown in Fig. 11, we see that this mode can extract the emission of both perpendicular dipoles and parallel dipoles oriented in the $x$ direction. On the other hand, the filtered TE mode extracts the emission of parallel dipoles oriented in the $z$ direction. Since dipoles located in the middle of the membrane couple to these modes very strongly, 

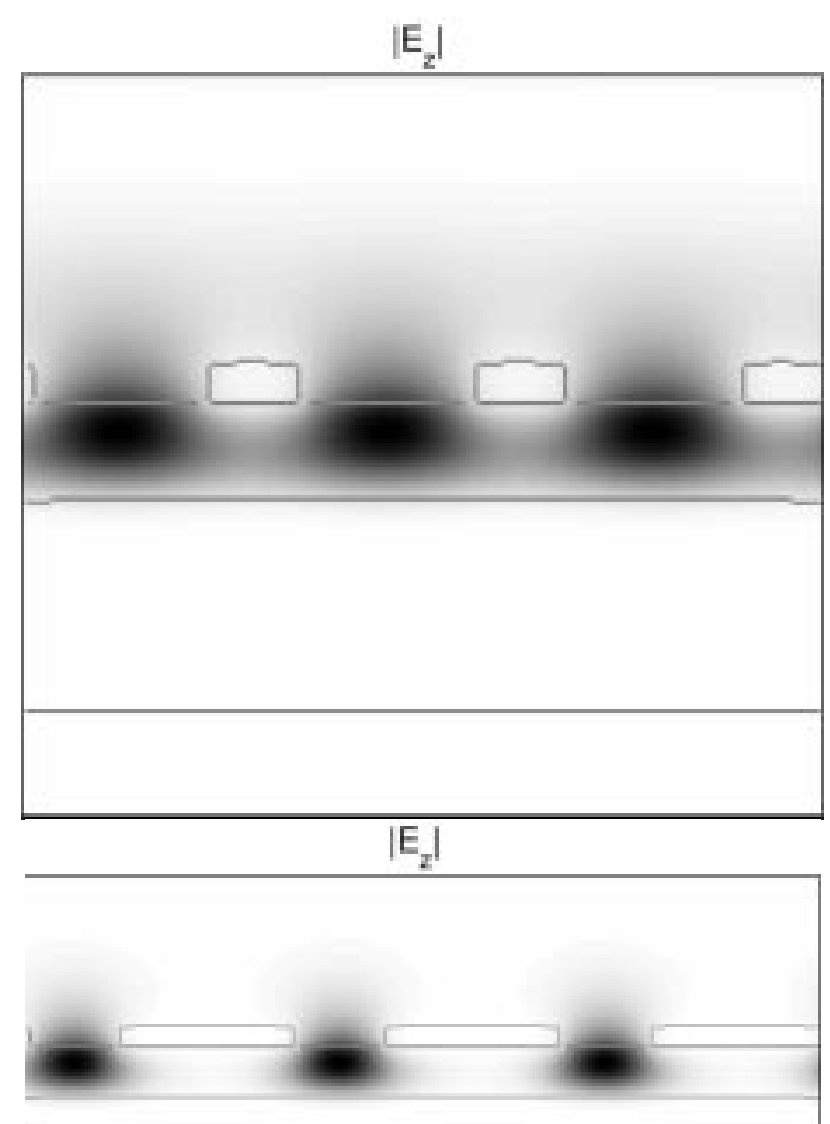

Fig. 13. $\left|E_{z}\right|$ of the TE mode with $k_{x}=0$ and $\lambda_{p} / \lambda=0.14$ for structures with periodicities of: (a) 250 and (b) $480 \mathrm{~nm}$.

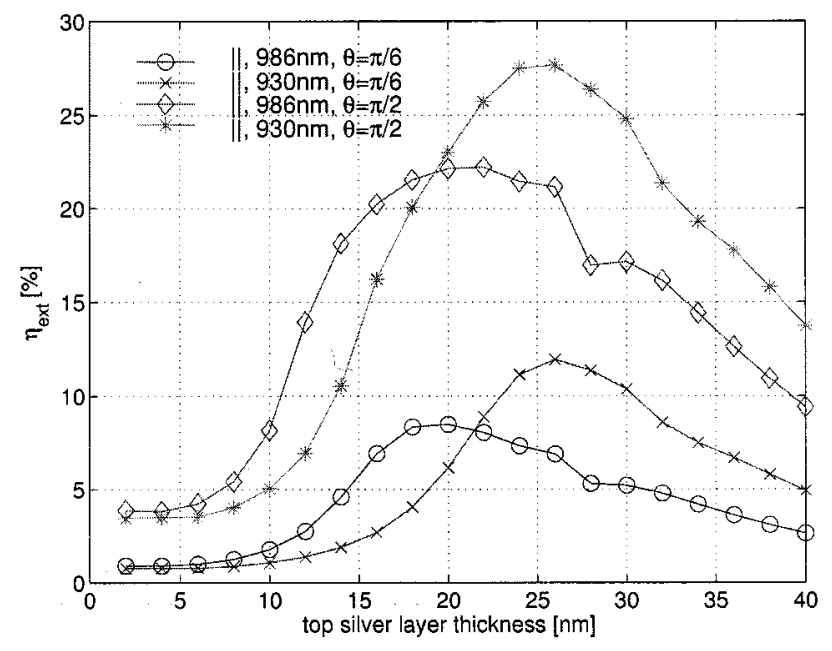

Fig. 14. Calculated external efficiency for the unpatterned metal clad microcavity, as a function of the top, semitransparent silver layer thickness. We calculated $\eta_{\text {cxt }}$ into the $30^{\circ}$ or $90^{\circ}$ collection angle. The emitter is a parallel dipole positioned in the middle of the $90-\mathrm{nm}$-thick membrane, oscillating at the wavelength of 986 or $930 \mathrm{~nm}$.

we expect that this structure will have a better external efficiency than the unpatterned structure.

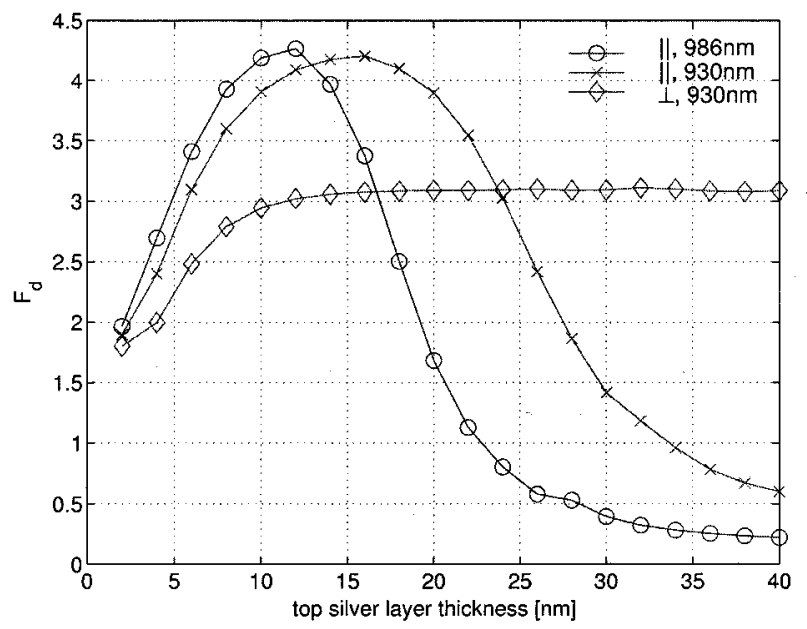

Fig. 15. Calculated decay rate enhancement for the unpatterned metal clad microcavity, as a function of the top, semitransparent silver layer thickness. The emitter is a parallel or a perpendicular dipole positioned in the middle of the 90-nm-thick membrane, oscillating at the wavelength of 986 or $930 \mathrm{~nm}$.

One should also note low values of $Q$ resulting from a significant metal absorption. A reduction in the absorption and a further increase in the extraction can be achieved by designing a device that operates at longer wavelengths. As we will see later, the calculated $Q$ values are in the same range as our experimental results.

\section{E. Purcell Factor and Decay Rate Enhancement}

The Purcell factor $\left(F_{p}\right)$ is defined as the spontaneous emission rate enhancement in a microcavity relative to a bulk semiconductor. On the other hand, we define the decay rate enhancement $\left(F_{d}\right)$ as a ratio of the total decay rate in a microcavity $(1 / \tau)$ and the spontaneous emission rate in a bulk semiconductor $\left(1 / \tau_{0}\right)$

$$
F_{d}=\frac{\frac{1}{\tau}}{\frac{1}{\tau_{0}}} .
$$

The modulation speed of an LED is proportional to $F_{d}$. When analyzing a light-emitting device, we care about the overall efficiency, defined as a product of $F_{d}$ and the external efficiency $\eta_{\text {ext }}$

$$
F_{d} \cdot \eta_{\text {ext }}=\frac{\frac{1}{\tau_{r}}}{\frac{1}{\tau_{0}}} .
$$

The efficiency is a measure of how much faster is the rate of light emission outside the cavity than the spontaneous emission rate in a bulk semiconductor.

We evaluated $F_{\boldsymbol{d}}$ analytically for unpatterned structures using the method described in [20]. The result is shown in Fig. 15. Subscripts $\|$ and $\perp$ denote parallel and perpendicular dipoles, respectively. If the excitation of surface plasmon modes is included in the spontaneous emission rate calculation 


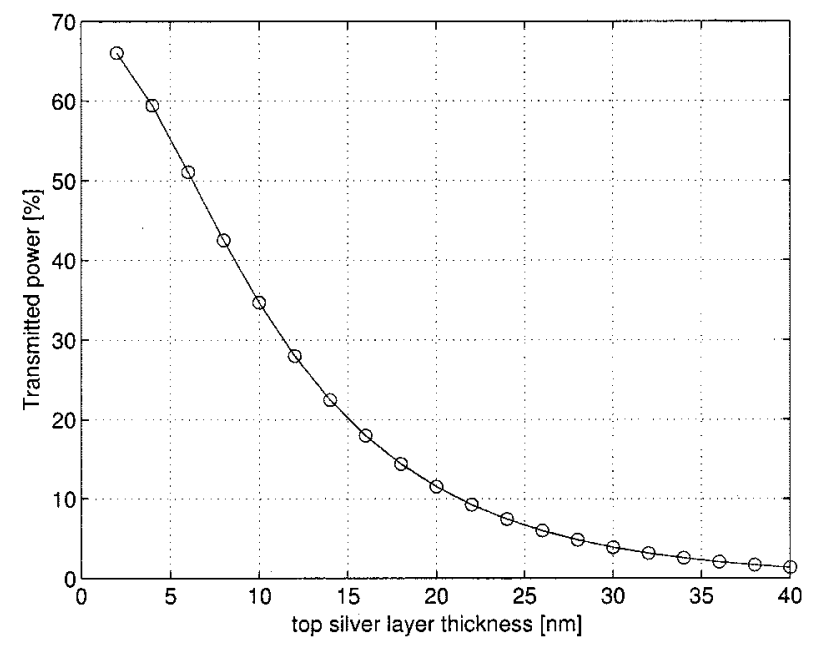

Fig. 16. Percentage of the pump beam intensity transmitted through the top, unpatterned silver layer, as function of its thickness.

for this structure, one obtains that $F_{d} \approx F_{p}$ for both parallel and perpendicular dipoles located in the middle of the membrane.

For complicated geometries obtained after the top metal layer patterning, it is possible to use the FDTD-based 3-D analysis of Purcell factor proposed in [22]. However, this requires large amounts of computer memory. Instead, it can be roughly assumed that decay rate enhancements and Purcell factors of fully processed, patterned structures have their values between those of the half-processed structure and the flat (unpatterned) metal clad microcavity.

\section{F. Pump Power Transmission Through the Top Surface}

In our experiments, we pump structures optically from top using a semiconductor laser diode emitting at $830 \mathrm{~nm}$. Therefore, it is important to determine what percentage of the $s$-polarized pump beam is transmitted into the structure.

Let us denote by $T$ the transmission through the top surface at the pump wavelength. $T$ can be evaluated from Fresnel equations for an unpatterned top layer. The calculated $T$, as a function of the silver layer thickness, is shown in Fig. 16. The transmission through the air/semiconductor interface is equal to $70 \%$. For structures with metallic grating on top, there is no increase in the pump power transmission due to diffraction at the patterned metal layer, and transmission coefficients can be approximated by weighting previously calculated coefficients with the filling factor of the grating. In order to prove that this is correct, we analyzed (using the 2-D FDTD method) the transmission through the metallic grating sitting on top of a semiconductor. The metal layer had the same parameters as the previously simulated grating with a periodicity of $250 \mathrm{~nm}$ and absorption losses were included. We analyzed one unit cell of the structure and applied Mur's ABC to boundaries in the $y$ direction and periodic boundary conditions (i.e., Bloch boundary conditions with $k_{x}=0$ ) to boundaries in the $x$ direction. A parallel dipole (oriented in the $x$ or $z$ direction) was located $400 \mathrm{~nm}$ above the metal surface, in the air and above the middle of the gap between stripes. The frequency of dipole oscillations matched the pump frequency. We calculated the power of the dipole source, as well as the power transmitted into the semiconductor, by integrating the Poynting's vector along a chosen surface. Without metal on top, the calculated transmission was $T=0.7$ for both $x$-oriented or $z$-oriented dipole, as expected from Fresnel equations. However, in the presence of the metallic grating, we calculated $T=0.5$ for an $x$-oriented dipole and $T=0.24$ for a $z$-oriented dipole. This means that the average transmission in the presence of a grating is $T=0.375$. By weighting transmission coefficients for the flat metal layer on top ( $40 \mathrm{~nm}$ thick) and for the air/semiconductor interface with the filling factor of the grating, we obtain $T=0.47$.

\section{G. Trapping of Pump Photons Within the Microcavity}

We can define $N$ as a factor that measures an increase in the pumping intensity resulting from the trapping of pump photons within the microcavity. $N$ is proportional to an average number of times that a pump photon crosses the QW before being absorbed in it or lost through other nonradiative loss mechanisms. The trapping of pump photons within the microcavity effectively increases the pumping intensity, since the probability that a photon excites an electron transition into an upper band is proportional to the number of times that it crosses the QW. The unprocessed wafer has $N=1$, since there is no mode within the structure that pump photons can couple to and photons not absorbed in the QW are lost when they reach the GaAs substrate. However, in the case of half-processed or fully processed wafers, the pump power transmitted through the top surface can couple to an $s$-polarized guided mode of the structure. For fully processed wafers, this can be easily seen from the previously calculated TE band diagrams (the pump wavelength is equal to 0.17 in units $\lambda_{p} / \lambda$ ). For the half-processed wafer, we performed the 1-D finite-difference analysis and showed that an $s$-polarized guided mode existed at the wavelength of $830 \mathrm{~nm}$.

Let us denote by $\alpha$ (in units [1/cm]) the loss coefficient for the guided mode that pump photons couple to. The angle with respect to the $y$ axis of this mode's total internal reflection is defined as

$$
\theta=\arcsin \left(\frac{k_{x}}{k}\right)
$$

where $k_{x}$ is the wavevector component in the direction of propagation $(x)$ and $k$ is the amplitude of the wavevector. Then, it follows that $N$ is proportional to

$$
N \cong \frac{1}{\alpha d \tan (\theta)}=\frac{\sqrt{k^{2}-k_{x}^{2}}}{\alpha d k_{x}}
$$

where $d$ is the membrane thickness. $N$ cannot be considered to be the exact value of the increase in the pumping intensity, since we arbitrarily chose to evaluate it until the trapped beam energy drops to $10 \%$ of its initial value. However, it is the indicator that the trapping happens and how strong it is. We analyze the half-processed wafer and the flat metal clad microcavity using the 1-D finite difference method (absorption losses in both metal and semiconductor were included, former being dominant). From the obtained values of $\alpha$ and $k_{x}$ for the guided TE mode at $830 \mathrm{~nm}$, we estimate $N$. The unpatterned metal clad microcavity has $N=12$, even when the top silver layer 


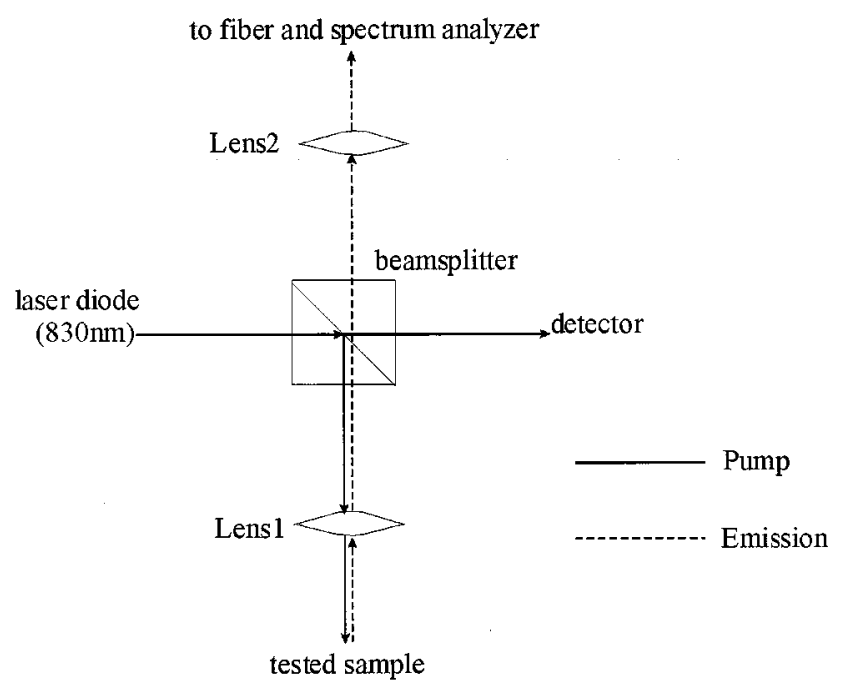

Fig. 17. Experimental setup used for PL measurements.

thickness varies between 20 and $40 \mathrm{~nm}$. For the half-processed structure, consisting of a lifted-off membrane on top of a thick bottom metal layer, we also estimate $N=12$. Therefore, in both structures, there is an equal increase in the pumping intensity resulting from the pump beam trapping in the microcavity.

\section{MEASUREMENTS}

The experimental setup used for the PL measurements is shown in Fig. 17. Samples are mounted on an X-Y-Z stage and optically pumped at a $90^{\circ}$ incident angle. The pump source is a semiconductor laser diode emitting at $830 \mathrm{~nm}$, pulsed with a period of $3 \mu \mathrm{s}$ and a pulse width of $2.5 \mu \mathrm{s}$. A nonpolarizing cube beamsplitter is used to bring $50 \%$ of the pump beam to the detector and to measure the external pump power. The other $50 \%$ of the beam is focused to a $10-\mu \mathrm{m}$ spot on the sample by using a 100X objective lens (labeled as Lens 1). The same objective lens is also used to collect the emitted light. The collection angle is $30^{\circ}$ with respect to normal. The collected emission is then focused in Lens 2 and detected using a fiber coupled spectrum analyzer.

We have measured the output from the unprocessed as-grown wafer, from the half-processed wafer, and from the fully processed structures, with a patterned or unpatterned thin metal layer on top. We fabricated a series of gratings in the top metal layer, that was $25 \mathrm{~nm}$ thick (within a $\pm 10 \%$ range). Each grating is characterized with a periodicity $(a)$ and a gap $(g)$ between silver stripes. Between stripes, silver was completely removed by $\mathrm{Ar}^{+}$ion milling. During the thin silver layer deposition, part of the sample surface was masked, in order to also produce half-processed regions. The measurement results are summarized in Table II, where the peak external pump power was 2.2 mW. $E_{\text {up }}(986 \mathrm{~nm})$ and $E_{\mathrm{hp}}(986 \mathrm{~nm})$ denote the PL enhancements at $986 \mathrm{~nm}$ of the fully processed wafer with respect to the unprocessed and half-processed wafers, respectively. Tabulated values are raw measured data and have not been rescaled. For all
TABLE II

PHOTOLUMINESCENCE MEASURED FROM THE FABRICATED STRUCTURES. $a$ IS the 1-D GRating Periodicity, While $g$ Denotes the Gap BetweEn Silver STRIPES. $E_{\text {up }}(986 \mathrm{~nm})$ AND $E_{\mathrm{l} p \mathrm{p}}(986 \mathrm{~nm})$ DENOTE PHOTOLUMINESCENCE ENHANCEMENTS AT $986 \mathrm{~nm}$ OF THE FULLY PROCESSED WAFER WITH RESPECT TO THE UNPROCESSED AND TO THE HALF-PROCESSEDWAFER, RESPECTIVELY. THE LAST ROW CORRESPONDS TO AN UNPATTERNED Top Metal Layer. All Tabulated Values Are Averaged OVER SEVERAL MEASUREMENTS

\begin{tabular}{c|c|c|c}
\hline$a[\mathrm{~nm}]$ & $g[\mathrm{~nm}]$ & $E_{u p}(986 \mathrm{~nm})$ & $E_{h p}(986 \mathrm{~nm})$ \\
\hline 250 & 160 & 46 & 2.24 \\
\hline 480 & 160 & 5.6 & - \\
\hline 650 & 160 & 6.4 & - \\
\hline 0 & 0 & 5.5 & - \\
\hline
\end{tabular}

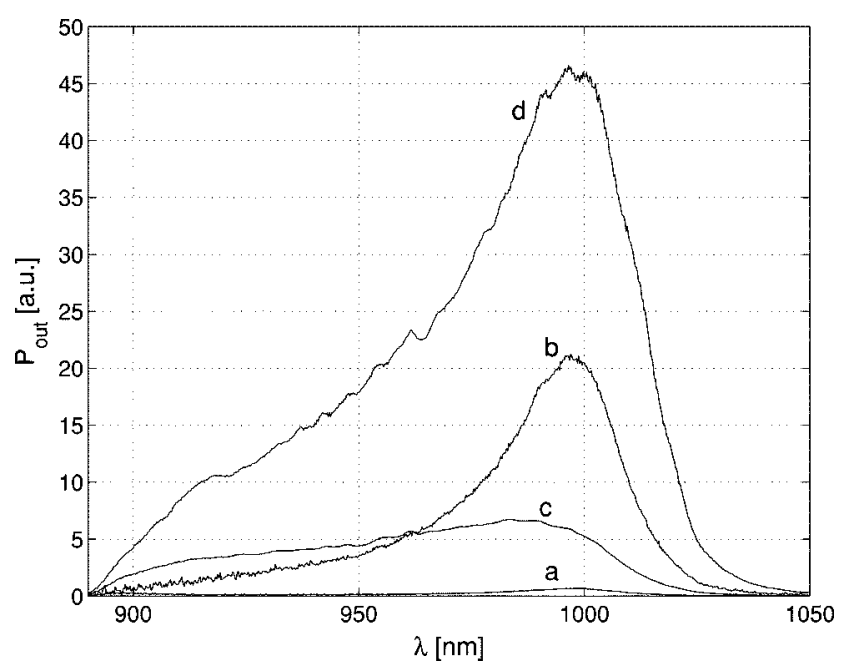

Fig. 18. The measured PL spectra: (a) unprocessed wafer; (b) half-processed wafer; (c) pattern with $a=650 \mathrm{~nm}$ and $g=160 \mathrm{~nm}$ (the unpatterned structure and the pattern with $a=480 \mathrm{~nm}$ and $g=160 \mathrm{~nm}$ give very similar signals); and (d) pattern with $a=250 \mathrm{~nm}$ and $g=160 \mathrm{~nm}$.

fully processed wafers, including the unpatterned metal clad microcavity, the FWHM is in the range of $60-110 \mathrm{~nm}$. Therefore, their quality factors are between 10-15. For the half-processed wafer, the FWHM is $32 \mathrm{~nm}$. Because of a bulk emission tail at lower wavelengths, a luminescence peak at $930 \mathrm{~nm}$ for unprocessed wafers cannot be clearly resolved. The spectra for unprocessed, half-processed and fully processed wafers are shown in Fig. 18. The PL peak of the unprocessed wafer at $986 \mathrm{~nm}$ was normalized to 1 . A filter was applied before the detection to cut off wavelengths below $890 \mathrm{~nm}$. A deposition of metal at one or at both sides of a 90-nm-thin semiconductor membrane does not seem to induce additional strain, since for all measured structures luminescence peaks are located at wavelengths of 986 and $930 \mathrm{~nm}$.

In order to explain measurement results, we have to take into account several effects: A) the increase in the pumping intensity resulting from the trapping of pump photons within a microcavity; B) the decay rate enhancement; and C) the change in the external efficiency. In our explanation, we will use theoretical results presented in the previous section of this article. The spontaneous emission reabsorption is not very efficient because of the small optical confinement factor in the QW and the photon recycling effect can also be neglected [3]. 
If $P_{\text {in }}$ denotes the external pump power and $P_{\text {out }}$ denotes the power emitted from the device, then

$$
\frac{P_{\text {out }}}{P_{\text {in }}} \approx \gamma
$$

where $\gamma$ is defined as

$$
\gamma=\beta \cdot\left(f_{\|} F_{d, \|} \eta_{\text {ext }, \|}+f_{\perp} F_{d, \perp} \eta_{\text {ext }, \perp}\right) .
$$

For patterned structures, the first term should be separated into contributions of parallel dipoles in the $x$ direction and in the $z$ direction, since they have different $\eta_{\text {ext }} . f_{\|}$and $f_{\perp}$ are fractions of dipole transitions that are in the $\mathrm{QW}$ plane or perpendicular to it. Their values at the main peak of $986 \mathrm{~nm}$ are $f_{\|}=1$ and $f_{\perp}=$ 0 and at $930 \mathrm{~nm}$ are $f_{\|}=1 / 3$ and $f_{\perp}=2 / 3$. Furthermore, $1 / 2$ of parallel dipoles are oriented in the $x$ direction and the other half in the $z$ direction. $\beta$ is the equivalent pumping intensity that can be expressed as

$$
\beta=T \cdot N
$$

Let us label the half-processed wafer by a subscript $h p$, the unprocessed wafer using a subscript up, and denote the PL enhancement by $E$. If the input pump powers are the same (i.e., $P_{\text {in }}$ equal), PL enhancements can be expressed as

$$
\begin{aligned}
& E_{\mathrm{hp}}=\frac{\gamma}{\gamma_{\mathrm{hp}}} \\
& E_{\mathrm{up}}=\frac{\gamma}{\gamma_{\mathrm{up}}} .
\end{aligned}
$$

The half-processed wafer has a 21 -fold PL intensity enhancement at $986 \mathrm{~nm}$ relative to the unprocessed wafer. This is a result of the increased external efficiency into the collection angle of $30^{\circ}$ (by a factor of 2), the decay rate enhancement of $F_{d, \|}=1.5$ and the higher pumping intensity resulting from pump photons trapping within the structure. Therefore, the pump trapping factor for the half-processed wafer is $N_{\mathrm{hp}}=7$.

The unpatterned metal clad microcavity has a sixfold PL intensity increase at $986 \mathrm{~nm}$ and the prominent peak at $930 \mathrm{~nm}$. Relative to the half-processed wafer, PL enhancements at 986 and $930 \mathrm{~nm}$ are equal to 0.26 and 1.5 , respectively. In order to explain this result, we calculated $\left(\gamma N_{\mathrm{hp}} / \gamma_{\mathrm{hp}} N\right)$. The result is shown in in Fig. 19. Parameters without a subscript refer to the unpatterned metal clad microcavity. We theoretically predicted that $N \cong N_{\mathrm{hp}}$. For the top silver layer thickness of $27 \mathrm{~nm}$, the theoretically estimated $E_{\mathrm{hp}}$ values agree with the experimentally observed ones. For this silver layer thickness, we have estimated theoretically $F_{d, \|}(986 \mathrm{~nm})=0.5, F_{d, \|}(930 \mathrm{~nm})=$ $2.1, \eta_{\text {ext, }, \|}(986 \mathrm{~nm})=6 \%$ and $\eta_{\text {ext }, \|}(930 \mathrm{~nm})=12 \%$ (into the $30^{\circ}$ collection angle). Therefore, the increase in the PL intensity has to be a result of an increase in the efficiency, defined as $F_{d} \cdot \eta_{\text {ext }}$, which is six times larger than in the unprocessed wafer at $986 \mathrm{~nm}$. Furthermore, the enhancement in the 930-nm peak comes from the Purcell enhancement and the improved extraction for parallel dipoles.

Is it possible that the enhanced peak at $930 \mathrm{~nm}$ is a result of the unpatterned structure pumped stronger than the half-processed one? Since the transmission coefficient $T$ of the unpatterned structure is at least ten times smaller than the one of the

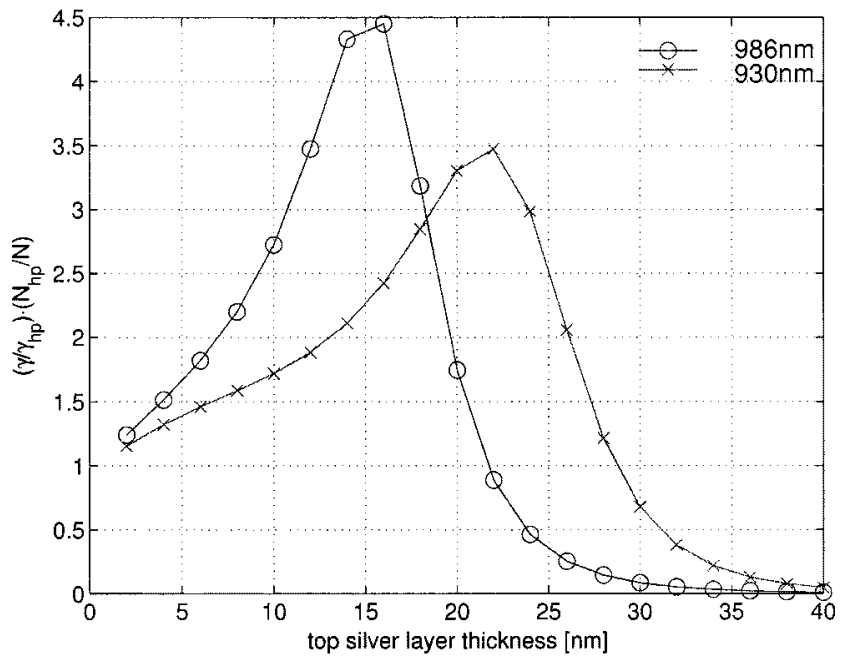

Fig. 19. Ratio of $\gamma$ factors for the unpatterned metal clad microcavity and the half-processed structure.

half-processed structure for the top silver layer thickness larger than $25 \mathrm{~nm}$, the stronger pumping of the unpatterned structure would require $N / N_{\mathrm{hp}}>10$. However, according to Fig. 19 and for the experimentally observed PL enhancement at $986 \mathrm{~nm}$, this would imply that $\left(\gamma N_{\mathrm{hp}} / \gamma_{\mathrm{hp}} N\right)<0.025$, which is possible only for thicker silver layers (thicker than $35 \mathrm{~nm}$ ). This not the case in our structure and, therefore, the enhanced peak at $930 \mathrm{~nm}$ cannot be a result of the strong pumping. In order to prove experimentally that the enhancement of the peak at $930 \mathrm{~nm}$ is not a result of the strong pumping, we measured the PL of the unpatterned microcavity at different pumping levels. The external pump power was controlled by neutral density filters. The shape of the PL spectra did not change when the pumping level was reduced.

Structures with periodicities of 480 or $650 \mathrm{~nm}$ have a similar performance to the unpatterned metal clad microcavity, as we theoretically predicted.

The structure with a periodicity of $250 \mathrm{~nm}$ has a 46-fold PL intensity enhancement at $986 \mathrm{~nm}$ relative to the unprocessed wafer. Clearly, we cannot determine exactly $T$ and $N$ factors for the patterned structure, but we can make a rough estimate and confirm that there is definitely an increase in the efficiency relative to the unprocessed wafer. Let us assume that the $N$ factor is of the same order as that of the half-processed wafer, and use the theoretically estimated value of the transmission coefficient $(T=40 \%)$. Then, we estimate that the pumping intensity has increased 4 times relative to the unprocessed wafer. This means that there is a 12-fold increase in the efficiency (into the detection angle of $30^{\circ}$ ) compared to the unprocessed wafer. The PL peak at $930 \mathrm{~nm}$ is not as large as in the unpatterned top layer case. The weaker peak at $930 \mathrm{~nm}$ can be explained by the fact that the Purcell enhancement at $930 \mathrm{~nm}$ is not as strong as in the unpatterned structure.

\section{CONCLUSION}

We have theoretically analyzed, fabricated, and measured the metal clad microcavity with a sub- $\lambda / 2$ semiconductor membrane and a patterned top metal layer. The emitting region is 
a single QW positioned in the middle of the membrane. At the same external pump power, we measure PL enhancements of up to 46 times with respect to the unprocessed wafer. We estimate that this enhancement is due to at least a 12-fold increase in the efficiency (relative to an unprocessed wafer), and an increase in the effective pumping intensity resulting from the pump photons trapping within the microcavity. Interesting modifications in the PL spectra were also experimentally observed, resulting from the simultaneous change in the spontaneous emission rate and the extraction efficiency.

Therefore, we showed that the coupling to surface plasmons in the patterned metal clad microcavity can be used to improve the light extraction and enhance the spontaneous emission rate in light-emitting devices. One of the advantages of this design is the small surface recombination rate, since the semiconductor is not perforated. Furthermore, metallic layers that are already included in the design can also be used as contacts for electrical pumping of the device. Certainly, in order to make good contacts, more attention has to be paid to the proper choice of metal. Devices presently operate in the 980-nm wavelength range, where metal absorption losses are significant. This reduces quality factors of fully processed structures to values between 10-15 and broadens their emission. This problem can be overcome by designing devices that operate at longer wavelengths. For example, a surface plasmon laser operating at $17 \mu \mathrm{m}$ has been demonstrated recently by researchers in Bell Labs [23].

We conclude that metal clad microcavities can be used as building blocks for highly efficient LEDs. However, in order to make a practical device, more research and work has to be done, particularly in designing their electrical properties.

\section{ACKNOWLEDGMENT}

The authors would like to thank O. Painter for the help with measurements and T. Yoshie for many useful suggestions.

\section{REFERENCES}

[1] I. Schnitzer, E. Yablonovitch, C. Caneau, T. J. Gmitter, and A. Scherer, " $30 \%$ external quantum efficiency from surface textured, thin film lightemitting diodes," Appl. Phys. Lett., vol. 63, no. 16, pp. 2174-2176, Oct. 1993.

[2] E. F. Schubert, N. E. J. Hunt, M. Micovic, R. J. Malik, D. L. Sivco, A. Y. Cho, and G. J. Zydzik, "Highly efficient light-emitting diodes with microcavities," Science, vol. 265, pp. 943-945, Aug. 1994.

[3] T. Baba, R. Watanabe, K. Asano, F. Koyama, and K. Iga, "Theoretical and experimental estimations of photon recycling effect in light emitting devices with a metal mirror," Jpn. J. Appl. Phys., vol. 35, pp. 97-100, Jan. 1996.

[4] A. Kock, E. Gornik, M. Hauser, and W. Beinstingl, "Strongly directional emission from AlGaAs/GaAs light-emitting diodes," Appl. Phys. Lett., vol. 57, no. 22, pp. 2327-2329, 1990.

[5] R. Windisch, P. Heremans, A. Knobloch, P. Kiesel, G. H. Dohler, B. Dutta, and G. Borghs, "Light-emitting diodes with $31 \%$ external quantum efficiency by outcoupling of lateral waveguide modes," Appl. Phys. Lett., vol. 74, no. 16, pp. 2256-2258, Apr. 1999.

[6] E. M. Purcell, "Spontaneous emission probabilities at radio frequencies," Phys. Rev., vol. 69, pp. 681-681, 1946.
[7] J. Gerard, B. Sermage, B. Gayral, B. Legrand, E. Costard, and V. TierryMieg, "Enhanced spontaneous emission by quantum boxes in a monolitic optical microcavity," Phys. Rev. Lett., vol. 91, no. 5, pp. 1110-1113, 1998.

[8] I. Gontijo, M. Boroditsky, E. Yablonovitch, S. Keller, U. K. Mishra, and S. P. DenBaars, "Coupling of InGaN quantum-well photoluminescence to silver surface plasmons," Phys. Rev. B, vol. 60, no. 16, pp. $11564-11567$, Oct. 1999.

[9] M. Boroditsky, R. Vrijen, T. F. Krauss, R. Coccioli, R. Bhat, and E. Yablonovitch, "Spontaneous emission extraction and purcell enhancement from thin-film 2-D photonic crystals," J. Lightwave Technol., vol. 17, pp. 2096-2112, Nov. 1999.

[10] W. L. Barnes, "Electromagnetic crystals for surface plasmon polaritons and the extraction of light from emissive devices," J. Lightwave Technol., vol. 17, pp. 2170-2182, Nov. 1999.

[11] L. A. Coldren and S. W. Corzine, Diode Lasers and Photonic Integrated Circuits. New York: Wiley, 1995.

[12] E. Yablonovitch, D. M. Hwang, T. J. Gmitter, L. T. Florez, and J. P. Harbison, "Van der Waals bonding of GaAs epitaxial liftoff films onto arbitrary substrates," Appl. Phys. Lett., vol. 56, no. 24, pp. 2419-2421, June 1990.

[13] S. A. Cummer, "An analysis of new and existing FDTD methods for isotropic cold plasma and a method for improving their accuracy," IEEE Trans. Antennas Propagat., vol. 45, pp. 392-400, Mar. 1997.

[14] J. B. Judkins and R. W. Ziolkowski, "Finite-difference time-domain modeling of nonperfectly conducting metallic thin-film gratings," $J$. Opt. Soc. Amer. B, vol. 12, no. 9, pp. 1974-1983, 1995.

[15] K. S. Yee, "Numerical solution to initial boundary value problems involving Maxwell's equations in isotropic media," IEEE Trans. Antennas Propagat., vol. AP-14, pp. 302-307, May 1966.

[16] A. Taflove, Computational Electrodynamics-The Finite-Difference Time-Domain Method. Norwood, MA: Artech House, 1995.

[17] O. Painter, J. Vučković, and A. Scherer, "Defect modes of a two-dimensional photonic crystal in an optically thin dielectric slab," J. Opt. Soc. Amer. B, vol. 16, no. 2, pp. 275-285, Feb. 1999.

[18] G. Mur, "Absorbing boundary conditions for the finite-difference approximation of the time-domain electromagnetic-field equations," IEEE Trans. Electromagn. Compat., vol. EMC-23, pp. 377-382, Nov. 1981.

[19] S. C. Kitson, W. L. Barnes, and J. R. Sambles, "Photonic band gaps in metallic microcavities," J. Appl. Phys., vol. 84, no. 5, pp. 2399-2403, 1998.

[20] J. A. E. Wasey and W. L. Barnes, "Efficiency of spontaneous emission from planar microcavities," J. Mod. Opt., vol. 47, no. 4, pp. 725-741, 2000.

[21] M. S. Tomaš and Z. Lenac, "Damping of a dipole in planar microcavities," Opt. Commun., vol. 100, no. 1-4, pp. 259-267, 1993.

[22] Y. Xu, J. S. Vučković, R. K. Lee, O. J. Painter, A. Scherer, and A. Yariv, "Finite-difference time-domain calculation of spontaneous emission lifetime in a microcavity," J. Opt. Soc. Amer. B, vol. 16, no. 3, pp. $465-474,1999$.

[23] A. Tredicucci, C. Gmachl, F. Capasso, and A. L. Hutchinson, "Single-mode surface-plasmon laser," Appl. Phys. Lett., vol. 76, no. 16 , pp. 2164-2166, 2000

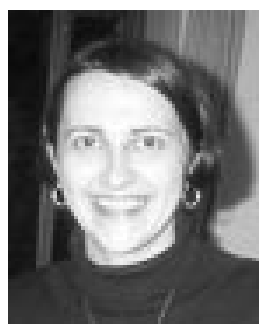

Jelena Vučković was born in Nišs, Yugoslavia, in 1971. She received the Dipl.Ing. degree from the Faculty of Electronic Engineering, University of Niš, Yugoslavia, in 1993. In 1997, she received the M.S. degree in electrical engineering from the California Institute of Technology, Pasadena, where she is currently working toward the Ph.D. degree.

From 1994 to 1995, she was with the University of Niš as an Assistant, and during 1996, she was with the School of Electrical Engineering, University of Sydney, Australia, as a Researcher. Her research interests include the design and fabrication of optical microcavities and photonic crystal-based optical devices. 


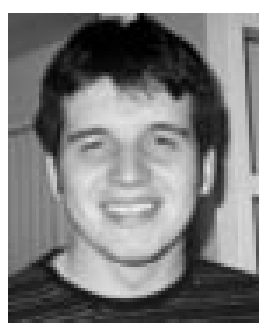

Marko Lončar was born in Dubrovnik, Croatia, in 1974. He has received the Dipl.Ing. degree from the Faculty of Electrical Engineering, University of Belgrade, Yugoslavia, in 1997, and the M.S. degree in electrical engineering from the California Institute of Technology, Pasadena, in 1998. Currently, he is working toward the Ph.D. degree in electrical engineering at the California Institute of Technology.

His research interests include design and fabrication of photonic crystal devices and ultrasmall device processing techniques.

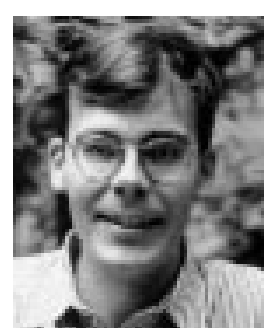

Axel Scherer received the B.S., M.S., and Ph.D. degrees from the New Mexico Institute of Mining and Technology,Socorro, in 1981, 1982, and 1985, respectively.

From 1985 until 1993, he worked in the Quantum Device Fabrication group at Bellcore. Currently he is Professor of Electrical Engineering, Applied Physics, and Physics at the California Institute of Technology, Pasadena, specializing in device microfabrication. His research interests include design and and microfluidic devices. 